Theories \& Applications, the International Edition

Printed Version: (ISSN 2090-5262)

Online Version: (ISSN 2090-5270)

July 2012, Volume 2, No. 2 Pages (117 - 127)

\title{
Energy Expenditure Related Differences Adaptations Following Resistance Training in Women
}

\author{
Nervana Ahmed Nasr Eddin*
}

\section{Abstract:}

Study aim: To assess the effects of Resistance training (RT) on resting metabolic rate, body composition and insulin sensitivity in women.

Material and methods: Two groups of women were studied. Group 1 (15 older women $60.60 \pm 3.09$ yr.; range 55- 65) and Group 2 (15 younger women $29.07 \pm 3.28$ yr.; range 25-35) non-obese women were engaged in resistance training $(R T)$ controlled in the same training session for trained 6-month, three times a week, 90 min per session, on non-consecutive days and exercised under the supervision of a personal trainer, to determine whether aging alters changes in fat-free mass (FFM), resting energy expenditure (REE), and glucose disposal.

Results: Our study showed that older \& younger women experienced a significant increase in fatfree mass (FFM), resting energy expenditure (REE), and glucose disposal following 6 months of resistance training (RT). Nevertheless, our results are similar to those of Lemmer et al. (2001) who found significant increases in FFM in younger and older women following RT although increases were greater in younger women.

Conclusions: older and younger women displayed an increase in FFM REE, and glucose disposal following 6 months of RT.

Keywords: Resistance training; Fat-free mass; resting energy expenditure (REE), and Glucose disposal rate; Pre\& Post menopausal women.

\section{Introduction:}

$\mathrm{C}$ ross-sectional studies conducted in females have shown an age-related decline of fatfree mass (FFM) and resting energy expenditure (REE) which appears to accelerate around age 65 yr (Pannemans and Westerterp, 1995; Poehlman et al., 1993). These alterations may lead to metabolic complications, such as decreased functional independence and deleterious changes in energy expenditure and glucose intolerance (Beaufrere and Morio, 2000).

Deleterious consequences related to the loss of muscle mass with advancing age (sarcopenia) could potentially be prevented by resistance

* Assistant Professor, Faculty of Physical Education for Girls, Helwan University, Egypt. training (RT) (Vincent et al., 2002; Poehlman, 2002; Hurley and Roth, 2000) as repeated bouts of RT are known to be a potent intervention for inducing muscle hypertrophy and potentially offset the aforementioned metabolic complications (Tipton, 2001). The concurrent training is widely used in fitness and physical condition programs, especially when the aim is to increase the energy expenditure during and after training session. However, it is not clear how the fatigue produced by a mode of exercise can adversely affect the quality and quantity of the other exercise mode (Lemos et al., 2009). While such metabolic adaptations in younger women have been well described (Poehlman and Melby, 1998; Poehlman et al., 2002; Cullinen and Caldwell, 1998), it is unclear if the aging process alters metabolic adaptations to RT. 
Few investigations have specifically examined potential age-related differences in metabolic adaptations. Some studies found RT to improve body composition, energy metabolism and glucose disposal in younger (Poehlman et al., 2000, 2002; Cullinen and Caldwell, 1998; Byrne and Wilmore, 2001) or in older women (Ryan et al., 1995; Fiatarone et al., 1990; Campbell et al., 1994), whereas other investigators obtained divergent results in varying age groups (Ivey et al., 2000; Lemmer et al., 2001).

One of the reasons that may explain these discrepancies is the use of different workloads in younger and older women. That is, it is possible that older women achieved a lower relative total workload compared to younger women and this could possibly explain age differences found in metabolic adaptations to chronic exercise. We examined age-related differences in metabolic adaptations following a RT training program as older and younger women participated in a relatively similar wellcontrolled RT. Direct measures of FFM, REE ((In body) and glucose disposal (hyperinsulinemic/euglycemic clamps) were performed prior and following the completion of the 6-month controlled RT program. Thereafter, we examined potential age related differences in body composition, energy expenditure and insulin sensitivity.

\section{Methods:}

\section{Subjects:}

15 older $60.60 \pm 3.09$ yr.; range 55- 65) and (21 younger women $29.07 \pm 3.28 \mathrm{yr}$.; range $25-35$ ) participated in this study. All subjects were healthy non-obese (BMI, $30 \mathrm{~kg} \mathrm{~m} 22$ ) women. Subjects were non-regular exercisers (2 times wk21) with stable weight $( \pm 2 \mathrm{~kg}$ during the 6month period preceding the study).

Exclusion criteria were: (1) previously diagnosed by medical authority to have diabetes, orthopedic limitations, pathologic fractures, and/or blood pressure at rest over $160 / 90 \mathrm{~mm} / \mathrm{Hg}$, (2) using hormone replacement therapy, over-the-counter medication, metabolism-alliterating substances (i.e. beta blockers, central alpha agonists, ace inhibitors, anti-arrhythmic, oral hypoglycemic agents, hypolipidemic agents, oral steroids, theophylline and xanthines). Subject

characteristics measured at baseline are listed in Table 1. For the younger group, all tests were performed during the luteal phase of the menstrual cycle. All subjects were informed of the risks associated with the procedures and all gave their written informed consent. The procedures were approved by the supervisor from Cairo University Faculty of Medicine. All participants were recruited by the use of an advertisement in local Health Club. An interview was conducted to screen for the aforementioned inclusion/exclusion criteria.

An eligibility visit was also held to confirm admissibility (i.e., physical examination, medical history). Two weeks after the screening visit, subjects were scheduled for a visit at Health Club. For the 3 previous days, participants were provided with a standardized diet prepared by the researcher containing 55\% carbohydrate, $25 \%$ fat, and $20 \%$ protein. Early morning, REE was measured followed by determinations of glucose disposal, VO2max and body composition. Upon successful completion of these tests, participants joined the RT program and were instructed to maintain body weight and usual lifestyle (i.e. day-to-day activities and diet). Once the exercise program was completed, subjects were submitted to postintervention metabolic tests identical to those of baseline examination. Follow-up testing was held; $72 \mathrm{~h} \pm 24 \mathrm{~h}$ after the subjects completed the program. This time lapse has been shown to be necessary to overcome the RT induced transient elevation in metabolism that follows a single resistive training bout (Tremblay et al., 1988).

Six younger women dropped out of the study at various points in time during the course of the study for the reasons of: non-compliance due to changes in work/school schedule, injury, health problems not related to study and refusal of post-testing. Regardless of the duration of their participation in the study, their data were excluded from the data set. Physical characteristics of drop-outs were not different from those of women who completed the study. None of women in the older cohort dropped out of the study. The net result was that 15 younger 
and 15 older women completed the entire training program.

\section{Resistance training program:}

Women trained three times a week on nonconsecutive days and exercised under the supervision of a personal trainer. The 1-RM (one-repetition-maximum) is defined herein as the heaviest weight that can be moved through the entire range of motion for no more than one repetition. To determine 1-RM, all subjects initially performed $3-5$ repetitions with a light weight to learn the proper technique. The trainer then selected a greater weight and asked the subject to perform the lift. After a 3-4 min rest, the next heaviest weight was lifted, and this was repeated until the subject could not complete the full lift. The 1-RM test was performed for the exercises of leg press, chest press, shoulder press, and seated cable rows at pre-, mid-, and post- combination resistance training (RT) for both age groups. These tests were used to measure and adjust workload during the program (preened mid-program) and measure strength gains (mid- and end-point).

All training sessions started with a warm-up consisting of $5 \mathrm{~min}$ of low-intensity cycling plus $10 \mathrm{~min}$ of static stretching that targeted all major muscle groups. Participants then performed the load phase of RT consisting in 3 10 repetitions for nine different muscle groups (leg press, chest press, leg extension, shoulder press, sit-ups, seated cable rows, triceps extensions, arm curls, and leg curls) aiming to provide a whole-body RT program. All exercises were performed on weight lifting machines and all sessions were individually monitored by a personal trainer to ensure wellcontrolled progression and optimal gains. Both younger and older women performed the same training program and were supervised by the same trainer throughout the program. The rate of attendance (three sessions of exercise/week with a maximum of one missed session/month accepted) for the workout sessions was similar for both cohorts. The load $(\mathrm{kg})$ was repeatedly adjusted to maintain the number of repetitions prescribed at baseline $(\mathrm{n}=10 ; 80 \% 1-\mathrm{RM})$. Resting periods of $60-90 \mathrm{~s}$ were taken between all sets.

\section{Body composition:}

Body Compassion was determined using (Body Compassion Instrument "In Body 230) with the volunteer in the fasting state and clothed in a physical gown. Height was measured using a tape measure fixed to the wall with the subject in stocking feet. Determination of fat mass and FFM was assessed using (Body Compassion In Body) (Starling et al., 1998 a,b). In lab of the laboratory of faculty of Physical Education for women- Helwan University, FFM is defined herein as the mass of tissue representing soft tissue exclusively (mineral body mass excluded).

\section{Resting energy expenditure:}

REE was measured by indirect calorimetry for $45 \mathrm{~min}$ as previously described (Tchernof et al., 1999), using a ventilated hood technique after a 12-h overnight fast at the Cardia-Respiratory treadmill. Respiratory gas analysis was performed using Cardia-Respiratory treadmill (Sensormedics, Yorba Linda, CA). REE (kcal d1) was calculated using the equation of Weir (Weir, 1949). The test-retest correlation coefficient for REE measured 1 week apart was 0.90 in our laboratory.

\section{Maximal oxygen consumption:}

VO2max was measured from an incremental treadmill test in which volunteers walked or ran to volitional exhaustion (Poehlman et al., 2000) using open-circuit spirometry. After a 3-min light warm-up, the grade of the motor-driven treadmill was incrementally increased by $2.5 \%$ every 2 min with the speed held constant. The criteria for the attainment of VO2max were: (1) a RER greater than 1.1 , (2) a heart rate at or above the age-predicted maximum, and (3) no further increase in oxygen uptake despite increasing workload. At least two of these criteria were met in all subjects. Test-retest analysis in nine individuals (tests underwent 1 week apart) yielded an intra-class correlation of 0.94 and a CV of $3.8 \%$ in our laboratory.

\section{Glucose disposal:}

Glucose disposal was determined using a hyperinsulinemic-euglycemic clamp according to DeFronzo et al. (1979) and as previously reported (Dvorak et al., 1999; Garcia-Rubi et al., 1998). Briefly, a teflon catheter was inserted into the antecubital vein for insulin and dextrose infusions. A second teflon catheter was inserted retrogradely into the dorsal vein of the 
contralateral hand for blood withdrawal during the clamp procedure. The subject's hand was placed in a hot box heated to $50 \mathrm{C}$ for sampling of arterialized blood. At min 0 , a continuous infusion of insulin was started at a constant rate (40 mU m2 body surface area min-1). A variables-peed infusion pump was used simultaneously for infusion of $20 \%$ dextrose attempting to level-off fasting glycemia (variation of no more than $5 \%$ from basal value). Blood samples were drawn every $5 \mathrm{~min}$ for glucose testing and served for insulin measurement at $-30,-10,0,30,60,75,90,105$, and $120 \mathrm{~min}$ of the clamp. The insulinstimulated glucose disposal rate (M-value) was calculated as the average dextrose infusion rate (mg min-1) during the last $30 \mathrm{~min}$ of the clamp.
Hepatic glucose production has been shown to be completely suppressed with the quantity of insulin used in the current study (Defronzo, 1979).

\section{Statistical analyses:}

Student's t-tests were performed to assess differences between age groups at baseline. Paired t-tests were used to examine the difference between baseline and follow-up measures in each group. Thereafter, $2 \times 2$ repeated measure ANOVA were used to examine the main effects of EXERCISE (pre- vs post-exercise) and AGE (younger vs older) on adaptations in FFM, REE and glucose disposal. Statistical analyses were performed using the SPSS software (version 16.0). 

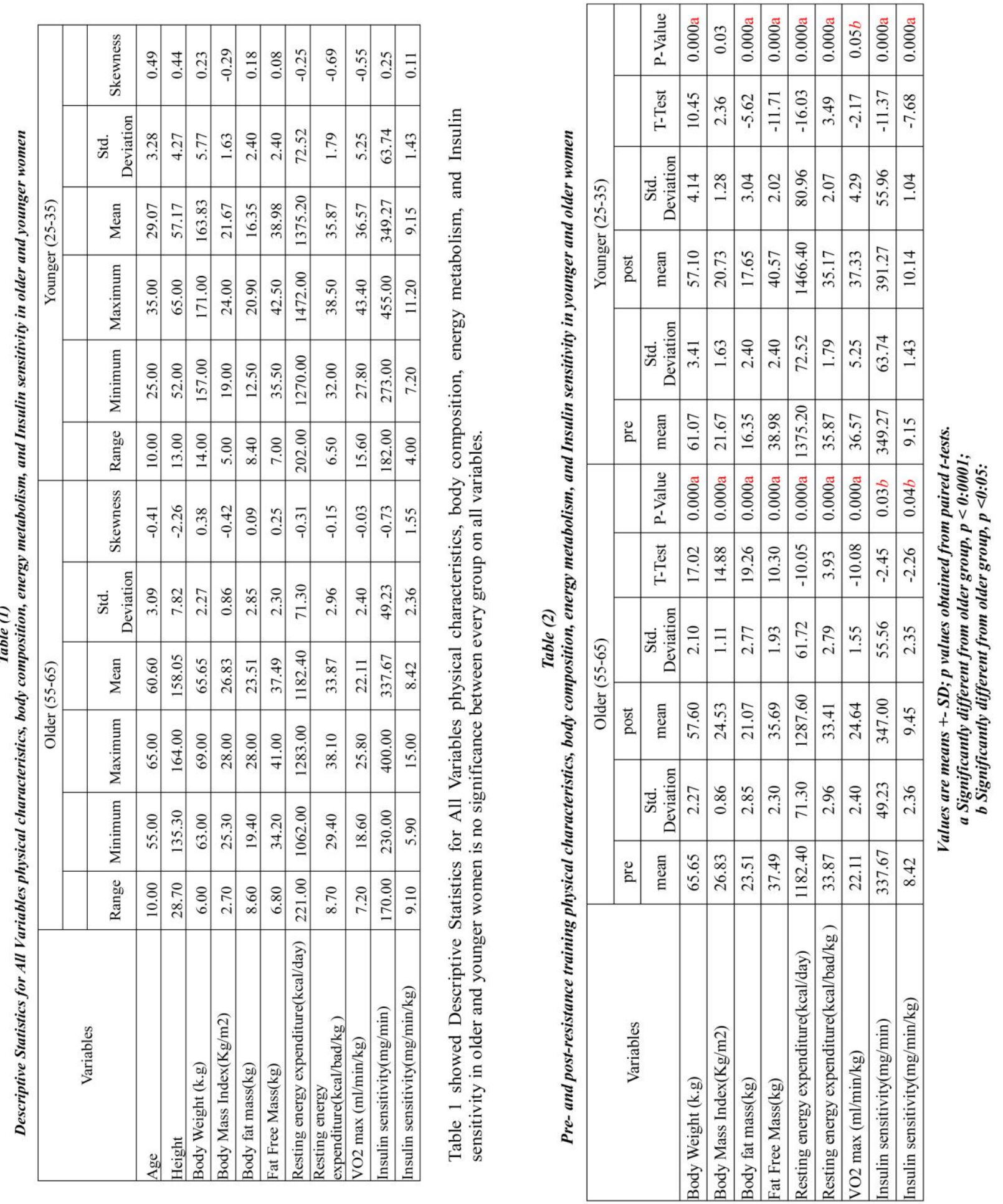


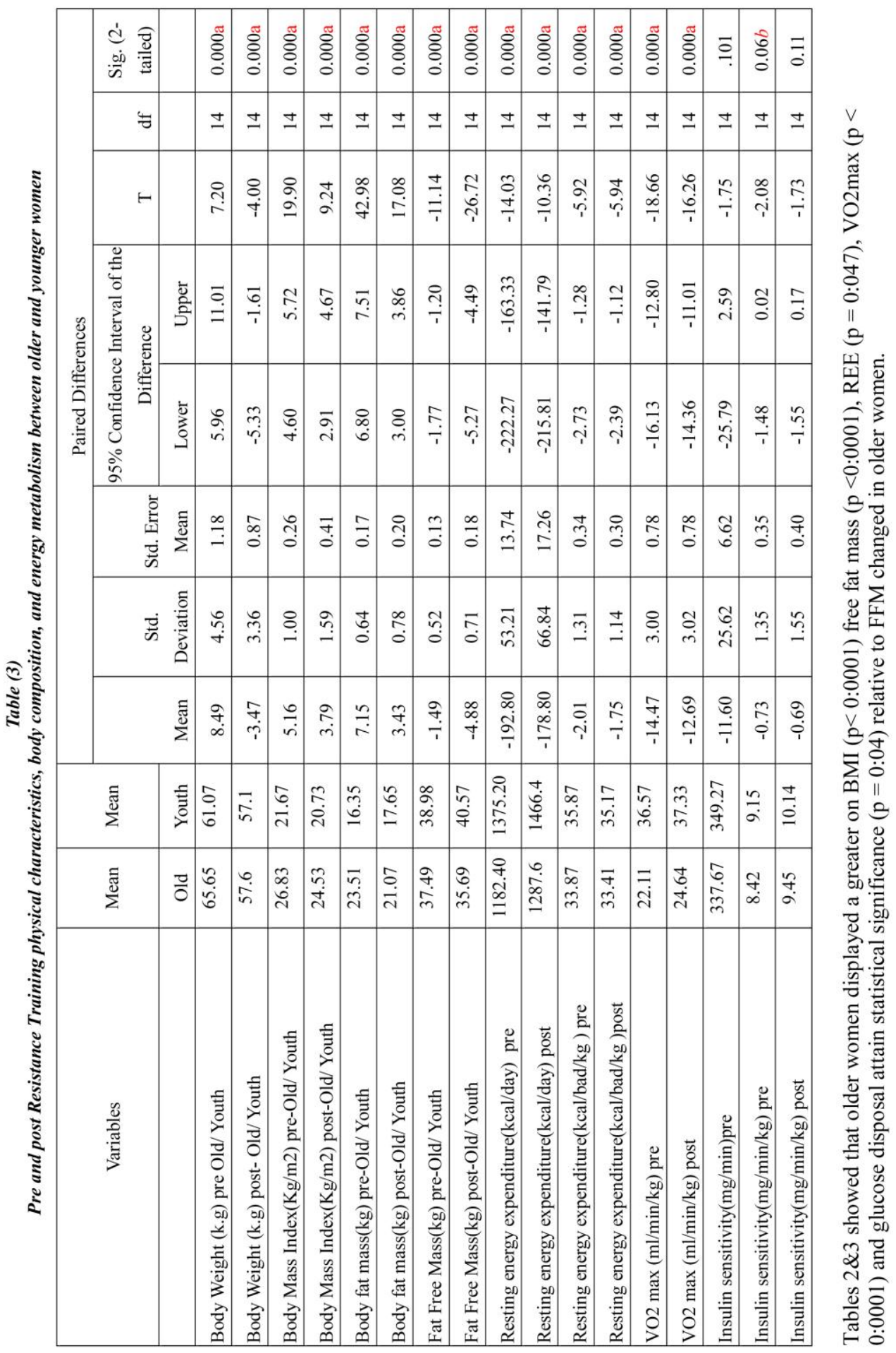




\section{Results:}

Fifteen older and younger women completed all the training sessions in addition to pre- and post-metabolic testing sequences. Subject characteristics are displayed in Table 1,2 and 3. Prior the beginning of training, Table 1 showed Descriptive Statistics for All Variables physical characteristics, body composition, energy metabolism, and Insulin sensitivity in older and younger women is no significance between every group on all variables. Tables $2 \& 3$ showed that older women displayed a greater on BMI ( $p<0: 0001)$ free fat mass ( $p<0: 0001)$, $\operatorname{REE}(\mathrm{p}=0: 047), \mathrm{VO} 2 \max (\mathrm{p}<0: 0001)$ and glucose disposal attain statistical significance $(\mathrm{p}$ $=0: 04)$ relative to FFM changed in older women. Older women also tended to show greater body weight $(\mathrm{p}=0: 000)$ despite having similar FFM following 6 months of RT.

Younger women significantly increased FFM $\mathrm{p}<0: 00$ ), absolute REE ( $\mathrm{p}=0: 000$ ), body weight $(\mathrm{p}=0: 00), \quad$ BMI $(\mathrm{p}, 0: 03)$, absolute glucose disposal $(p=0: 00)$, fat mass $(p=0.00)$ VO2max, glucose disposal relative to FFM changed in younger females. On the other hand, older women showed a decrease in body fat mass $(\mathrm{p}<$ 0:00) and a trend toward an increase in FFM (p $=0: 000$ ), whereas body weight, BMI, REE, VO2max and glucose disposal (absolute or relative to FFM) remained changed.

Additional analyses showed that there was an age effect in the response of REE following RT even when the change in FFM following RT was used as a cavort. This suggests that the age difference observed in REE alteration following RT is not solely explained by the greater increase in FFM in younger than older women. However, no such age effect was found for glucose disposal although it was significantly increased in younger women and in older women following RT. This suggests that age does not affect glucose disposal response to RT but it is possible that a lack of statistical power may have impaired our capacity to show significant increases in glucose disposal in older women.

Muscular strength (1-RM) increased significantly during the course of the program in both age groups. The gains in strength relative to baseline values following 24 wk of RT were similar between younger and older women (leg press: 34 vs 29\%; chest press: 43 vs $41 \%$; rowing: 30 vs $26 \%$; shoulder press: 29 vs $27 \%$, respectively; all n.s.). This reinforces the fact that the relative workload of the RT program was well-controlled and similar in both age groups. Thus, any age-difference found in metabolic adaptations following RT is not likely to be attributed to a lower relative exercise intensity in older women.

\section{Discussion:}

There is a paucity of studies (Ivey et al., 2000; Lemmer et al., 2001) that directly compared RT-induced metabolic changes in body composition and energy metabolism in younger and older women. 1. Moreover, a recent metaanalysis (Toth et al., 1999) showed that approximately $50 \%$ of the studies reported no meaningful effects of RT on FFM in older persons. Although methodological issues may partly explain these divergences (Toth et al., 1999), other determinants are likely to contribute to the broad range of findings in the literature (e.g. the design of the training regimen, training modalities such as intensity of muscular contractions, or the number of repetitions per series). Thus, at this point, the quantity and volume of RT required to improve body composition and metabolic function in older women is not well understood.

We investigated the effects of a 6-month controlled RT program on body composition, energy metabolism, and glucose disposal in both younger and older women. We provided a relatively similar training stimulus to younger and older women. That is, we compared RTinduced metabolic alterations following a RT program that was similar in younger and older women for frequency, duration of the program, relative intensity, and number of sets and repetitions performed. RT increased FFM, glucose disposal, and REE in the younger group whereas the older group tended to increase FFM, lose fat mass and showed significant change in REE and glucose disposal. These results, although preliminary, may suggest an age-related resistance to alterations in metabolic function in response to chronic RT. 
Our study showed that younger women experienced a significant increase in FFM following 6 months of RT and older women tended to show such an increase. Nevertheless, our results are similar to those of Lemmer et al. (2001) who found significant increases in FFM in younger and older women following RT although increases were greater in younger women. On the other hand, Ivey et al. (2000) did not find any increase in FFM neither in younger nor older women which may be partially due to the shorter duration of their exercise program ( 9 weeks).

Interestingly, older women increase REE as hypothesized, despite a tendency to increase FFM. These results are in agreement with previous findings of Lemmer et al. (2001) who suggested an effect of gender on RT-induced increase in REE. In this study, Lemmer et al. (2001) found a significant increase in REE following RT in older men only which is consistent with other studies (Hurley and Roth, 2000). In fact, RT-induced elevations in REE have been found to be significantly greater in males than in females (Cullinen and Caldwell, 1998; Lemmer et al., 2001), even when expressed per kilogram FFM (Lemmer et al., 2001), suggesting a sex-related difference. Although not measured in this study, several mechanisms could be suggested to potentially explain this gender difference. For instance, norepinephrine secretion in response to heavy resistive stimuli has been demonstrated to be unchanged in postmenopausal women following progressive RT (Ryan et al., 1995) whereas other investigators noted increased plasma norepinephrine levels in 50- to 65-yr-old men (Pratley et al., 1994). In women, the lack of response from the sympathetic nervous system may be involved in the difference of change in REE following RT.

An additional potential explanation for the lack of change in REE pertains to the loss of fat mass during RT in older women, which caused total body weight to remain similar. A diminution in total body weight, irrespective of changes in FFM, can potentially decrease REE as previously shown (Lemmer et al., 2001). Actually, among studies where increases in REE were noted, substantial increases in FFM and body weight were observed (Taaffe et al.,
1995). On the other hand, when RT did not produce changes in FFM, REE remained unchanged (Ryan et al., 2001). This may partially explain why older women failed to display an increased REE despite a trend to increase FFM following RT.

Our results also show that significant improvements in glucose disposal occurred in younger bigger than older women although further analyses indicated that the changes were not statistically different between the two groups. Whole-body glucose disposal has been shown to be increased after RT in younger women (Poehlman et al., 2000) and our results support this finding. However, it has been suggested that although older women may improve insulin release to a given amount of exogenous glucose following RT, this had no effect on glucose utilization, suggesting no improvement in glucose disposal (Joseph et al., 2001). In fact, previous studies have reported no improvements in glucose utilization following RT in older women (Brochu et al., 2002; Hughes et al., 1995). However, to our knowledge, no studies have compared changes in glucose disposal in response to RT between younger and older women when using similar RT programs. Since muscle is the most important tissue using glucose, it seems that an increase in FFM improve glucose disposal. Again, larger gains in FFM may be needed to produce such changes and this deserves further investigation.

Several limitations should be noted in the present study. First, the sample size cohort was relatively small and this may have impaired the statistical power to reveal significant increases in FFM $(\mathrm{n}=15)$ and in glucose disposal. Also, 1-RM testing protocol could possibly have under- evaluated the true individual maximal strength in older women (Braith et al., 1993; Sale, 1987), which would have affected the true relative workload and therefore minimizing FFM gains.

Nonetheless, this study provides several noteworthy clinical implications. For instance, RT may be considered an appropriate intervention to increase REE in premenopausal women and possibly improve insulin resistance in younger and older women. Together, these exercise-induced improvements support the 
theory that RT may be effective to offset obesity-related comorbidities as well as beneficial effects on strength and physical function that have been previously reported in older women (Ivey et al., 2000; Lemmer et al., 2001).

\section{Conclusion:}

older and younger women displayed an increase in physical characteristics, body composition, energy metabolism, and Insulin sensitivity following 6 months of RT. These results appeared to be independent of the gains in all variables. These results suggest an age-related alteration in metabolic changes in response to a 6-month RT program in previously untrained women.

\section{References:}

1. Beaufrere, B., Morio, B., 2000. Fat and protein redistribution with aging: metabolic considerations. Eur. J. Clin. Nutr. 54 (Suppl. 3), S48-S53.

2. Braith, R.W., Graves, J.E., Leggett, S.H., Pollock, M.L., 1993. Effect of training on the relationship between maximal and submaximal strength. Med. Sci. Sports Exerc. 25 (1), 132 138.

3. Brochu, M., Savage, P., Lee, M., Dee, J., Cress, M.E., Poehlman, E.T., et al., 2002. Effects of resistance training on physical function in older disabled women with coronary heart disease. J. Appl. Physiol. 92 (2), 672-678.

4. Byrne, H.K., Wilmore, J.H., 2001. The effects of a 20 -week exercise training program on resting metabolic rate in previously sedentary, moderately obese women. Int. J. Sport Nutr. Exerc. Metab. 11 (1), 15-31.

5. Campbell, W.W., Crim, M.C., Young, V.R., Evans, W.J., 1994. Increased energy requirements and changes in body composition with resistance training in older adults. Am. J. Clin. Nutr. 60 (2), 167-175.

6. Cullinen, K., Caldwell, M., 1998. Weight training increases fat-free mass and strength in untrained young women. J. Am. Diet Assoc. 98 (4), 414-418.
7. Defronzo, R.A., 1979. Glucose intolerance and aging: evidence for tissue insensitivity to insulin. Diabetes 28 (12), 1095-1101.

8. DeFronzo, R.A., Tobin, J.D., Andres, R., 1979. Glucose clamp technique: a method for quantifying insulin secretion and resistance. Am. J. Physiol. 237 (3), E214-E223.

9. Dvorak, R.V., DeNino, W.F., Ades, P.A., Poehlman, E.T., $1999 . \quad$ Phenotypic characteristics associated with insulin resistance in metabolically obese but normal-weight young women. Diabetes 48 (11), 2210-2214.

10. Fiatarone, M.A., Marks, E.C., Ryan, N.D., Meredith, C.N., Lipsitz, L.A., Evans, W.J., 1990. High-intensity strength training in nonagenarians. Effects on skeletal muscle. JAMA 263 (22), 3029-3034.

11. Garcia-Rubi, E., Starling, R.D., Tchernof, A., Matthews, D.E., Walston, J.D., Shuldiner, A.R., et al., 1998. Trp64Arg variant of the beta3-adrenoceptor and insulin resistance in obese postmenopausal women.

12. J. Clin. Endocrinol. Metab. 83 (11), 40024005. Hughes, V.A., Fiatarone, M.A., Fielding, R.A., Ferrara, C.M., Elahi, D., Evans, W.J., 1995. Long-term effects of a highcarbohydrate diet and exercise on insulin action in older subjects with impaired glucose tolerance. Am. J. Clin. Nutr. 62 (2), 426-433.

13. Hurley, B.F., Roth, S.M., 2000. Strength training in the elderly: effects on risk factors for age- related diseases. Sports Med. 30 (4), 249-268.

14. Ivey, F.M., Roth, S.M., Ferrell, R.E., Tracy, B.L., Lemmer, J.T., Hurlbut, D.E., et al., 2000. Effects of age, gender, and myostatin genotype on the hypertrophic response to heavy resistance strength training. J. Gerontol. A Biol. Sci. Med. Sci. 55 (11), M641 M648.

15. Joseph, L.J., Trappe, T.A., Farrell, P.A., Campbell, W.W., Yarasheski, K.E., Lambert, C.P., et al., 2001. Short-term moderate weight loss and resistance training do not affect insulin-stimulated glucose disposal in postmenopausal women. Diabetes Care 24 (11), 1863-1869. 
16. Lemmer, J.T., Ivey, F.M., Ryan, A.S., Martel, G.F., Hurlbut, D.E., Metter, J.E., et al., 2001. Effect of strength training on resting metabolic rate and physical activity: age and gender comparisons. Med. Sci. Sports Exerc. 33 (4), 532-541.

17. Pannemans, D.L., Westerterp, K.R., 1995. Energy expenditure, physical activity and basal metabolic rate of elderly subjects. Br. J. Nutr. 73 (4), 571-581.

18. Poehlman, E.T., 2002. Menopause, energy expenditure, and body composition. Acta Obstet. Gynecol. Scand. 81 (7), 603-611. Poehlman, E.T., Melby, C., 1998. Resistance training and energy balance. Int. J. Sport Nutr. 8 (2), 143-159.

19. Poehlman, E.T., Goran, M.I., Gardner, A.W., Ades, P.A., Arciero, P.J., KatzmanRooks, S.M., et al., 1993. Determinants of decline in resting metabolic rate in aging females. Am. J. Physiol. 264 (3 Pt 1), E450E453.

20. Poehlman, E.T., Dvorak, R.V., DeNino, W.F., Brochu, M., Ades, P.A., 2000. Effects of resistance training and endurance training on insulin sensitivity in nonobese, young women: a controlled randomized trial.

21. J. Clin. Endocrinol. Metab. 85 (7), 2463 2468. Poehlman, E.T., Denino, W.F., Beckett, T., Kinaman, K.A., Dionne, I.J., Dvorak, R., et al., 2002. Effects of endurance and resistance training on total daily energy expenditure in young women: a controlled randomized trial. J. Clin. Endocrinol. Metab. 87 (3), 1004-1009.

22. Pratley, R., Nicklas, B., Rubin, M., Miller, J., Smith, A., Smith, M., et al., 1994. Strength training increases resting metabolic rate and norepinephrine levels in healthy 50- to 65yr-old men. J. Appl. Physiol. 76 (1), 133-137.

23. Ryan, A.S., Pratley, R.E., Elahi, D., Goldberg, A.P., 1995. Resistive training increases fat- free mass and maintains RMR despite weight loss in postmenopausal women. J. Appl. Physiol. 79 (3), 818-823.

24. Ryan, A.S., Hurlbut, D.E., Lott, M.E., Ivey, F.M., Fleg, J., Hurley, B.F., et al., 2001. Insulin action after resistive training in insulin resistant older men and women. J. Am.

Geriatr.
25. Sale, D.G., 1987. Influence of exercise and training on motor unit activation. Exerc. Sport Sci. Rev. 15, 95-151. Starling, R.D., Toth, M.J., Carpenter, W.H., Matthews, D.E., Poehlman, E.T., 1998a. Energy requirements and physical activity in free-living older women and men: a doubly labeled water study. J. Appl. Physiol. 85 (3), 1063- 1069.

26. Starling, R.D., Toth, M.J., Matthews, D.E., Poehlman, E.T., 1998b. Energy requirements and physical activity of older free-living African- Americans: a doubly labeled water study. J. Clin. Endocrinol. Metab. 83 (5), 15291534.

27. Taaffe, D.R., Pruitt, L., Reim, J., Butterfield, G., Marcus, R., 1995. Effect of sustained resistance training on basal metabolic rate in older women. J. Am. Geriatr. Soc. 43 (5), 465471.

28. Tchernof, A., Starling, R.D., Walston, J.D., Shuldiner, A.R., Dvorak, R.V., Silver, K., et al., 1999. Obesity-related phenotypes and the beta3adrenoceptor gene variant in postmenopausal women. Diabetes 48 (7), 1425-1428.

29. Tipton, K.D., 2001. Muscle protein metabolism in the elderly: influence of exercise and nutrition. Can. J. Appl. Physiol. 26 (6), 588-606.

30. Toth, M.J., Beckett, T., Poehlman, E.T., 1999. Physical activity and the progressive change in body composition with aging: current evidence and research issues. Med. Sci. Sports Exerc. 31 (11 Suppl.), S590S596.

31. Tremblay, A., Nadeau, A., Fournier, G., Bouchard, C., 1988. Effect of a three-day interruption of exercise-training on resting metabolic rate and glucose-induced thermogenesis in training individuals. Int. J. Obes. 12 (2), 163-168.

32. Vincent, K.R., Braith, R.W., Feldman, R.A., Magyari, P.M., Cutler, R.B., Persin, S.A., et al., 2002. Resistance exercise and physical performance in adults aged 60 to 83 . J. Am. Geriatr. Soc. 50 (6), 1100-1107.

33. Weir, J.B., 1949. New methods for calculating metabolic rate with special reference to protein metabolism. J. Physiol. 109, 1-9. Soc. 49 (3), 247-253. 
34. Wojtek, C. Z., et al., 2011. Department of kinesiology and community health University of Illinois at Urbana-Champaign. ASCM position stand on exercise and physical older adults. activity for 